\title{
An Improved Semantic Web-based Search Model for Ecommerce using OWL2
}

\author{
B. Pam \\ Department of Computer Science \\ Federal College of Education \\ Pankshin, Plateau State, Nigeria
}

\author{
A. A. Obiniyi \\ Department of Computer Science \\ Faculty of Physical Science, \\ Ahmadu Bello University, Zaria \\ Nigeria
}

\author{
O. N. Oyelade \\ Department of Computer Science, \\ Faculty of Physical Science, \\ Ahmadu Bello University, Zaria
}

\begin{abstract}
Information sharing across independent web applications seems impossible with just the static world wide web applications. Meanwhile, with the advent of the semantic web, web applications interoperability through information sharing and Linked Data have been achieved. This interoperability is usually presented as a decentralized system that allows agents to crawl over them for information sharing or gathering, and is employed in developing web applications like ECommerce. However, the existing e-Commerce model is being limited by its knowledge representation language. This model employs ontological approach using OWL for knowledge modeling and representation. Hence, this research seeks to create an improved e-commerce ontological base model which has a richer and expressive knowledge base with OWL2 for knowledge modeling and representation. This knowledgebase will be compared against the e-Commerce knowledge base that was modeled with OWL. Search results from the new knowledge base by the user yielded a more specific and needful outcome as compared to search on the knowledge base created with OWL.
\end{abstract}

\section{Keywords}

E-commerce, OWL, OWL2, RDF, RDFS, SQWRL, and SPARQL

\section{INTRODUCTION}

The name given to the first web is static web because it allowed users to just click links in order to view information. This static Web platform help improved electronic commerce (E-commerce). However, the static web was not machined readable [1]. With the coming of the Semantic Web and its supporting technologies it has further improved the Web, which have enhanced E-commerce. Some of the Semantic Web languages are: XML, RDF/RDFS, OWL, OWL2 and also, query and rule languages like SPARQL, SQWRL and SWRL. In this research, the goal is to develop a data model with OWL2 and used SQWRL as a query tool for products search in B2C scenario. The remaining part of this paper discusses the related works, the proposed model and ontology development. Finally, the implementation of the E-Commerce application is discussed and then the experimental results.

\section{RELATED WORK}

[2] presents a framework for an ontology-based e-commerce product information retrieval system and proposes an ontology-based adaptation of the classical Vector Space Model with the consideration of the weight of product attribute. A components related ontology was built, which is adopted to annotate the html documents and construct concept vectors of the documents. However, the research could not uniquely identify every product modeled in the ontology: OWL does not provide this construct. The product search query pattern requires a serialization of OWL to RDF since SPARQL was used. [3], models an ecommerce data structure; a product data model to amplify the vision of Semantic Web-based eCommerce and defines the Ethiopian export products and services ontology (eeps) ontology that covers the representational needs of Ethiopia export business scenarios for commodity products and commodity services. He analyze the ecommerce domain, critically analyze and evaluate emerging technologies employed for Ecommerce, the experimentally analyze conceptual discovery of GoodRelations ecommerce ontology usage pattern. He designed a GoodRelations compliant bilingual ontology for export business domain and implemented the semantic based query with SPARQL. However, the semantic web query mechanism used misses out some information modeled in their expressive knowledge base, hence, reducing product search. [4], Compares the effectiveness of using SQWRL over SPARQL. Proofs that modeling B2C knowledgebase using OWL is more expressive than RDF/RDFS and they developed and implemented a semantic web based framework for B2C, however, this research could not model the E-Commerce knowledgebase to show that a product cannot be sold by a retailer. [6] proposed a generic approach to transform the existing web contents related to tourism domain into semantic web format using RDF with ontology. The system has three modules for converting unstructured tourism data into RDF, the first module handles the downloading of unstructured text using online search. It extracts heterogeneous tourism related text based on the keyword specified in the online search dialogue by linking the Google search engine to get the documents in HTML format. The second module Converting HTML into XML, by using a HtmlConvertor which in turn uses SAX parser to read HTML data and converts to XML. The next module is the Mapping Process. The XML documents are mapped an ontological based RDF file called Travel2.owl which contains the travel related information in turns of classes, instances, sub instances, object property, relation, data property and general axioms. When the travel related heterogeneous unstructured data from various web sites are extracted and converted into semantic web format called RDF. They are implemented in java. [7] worked on models and rules for customer on-boarding - a move the customer's IT environment into the service provider's mode of operation. The alluded that in shifting the customer's IT environment to the service provider's side, there are bound to be different scenarios from varying customers that are going to arrive at the service provider's side. Hence, there arises a 
need for automating the process variant configuration of each customer on the service provider's side.

As a result, they adopted the use of ontology and rules to both model the standard on-boarding process and configure process variants based on the business context that characterizes various scenarios and the semantic rules model adaptation policies and help generate a customized process variant schema on the fly.

\section{THE ARCHITECTURE OF THE PROPOSED MODEL}

The model being proposed by this paper is hereby presented in Figure 1, and following paragraphs briefly explains the components of the figure. The client layer serves as front-end for the users of the system which is the online buyers. The client layer provides the users with two user interactive interface. These are the query entry interface and the result display interface. Users or buyers are expected to search for their desired product through the query entry. The query entry interface accepts inputs in an English-like manner. This query is comparable to that of typing search request into the Google search engine. However, this proposed system seeks to convert the English-like query into a semantic web understandable query. This will enable the efficient querying of the ontological knowledgebase.

The query generator automatically composes a semantic web query using the Semantic Query Web Rule Language (SQWRL) based on the semantic web tokens sent to it. The work of the query generator interfaces between the semantic webs based ecommerce search engine and the users end. The query generated by this component is further sent to the controller.

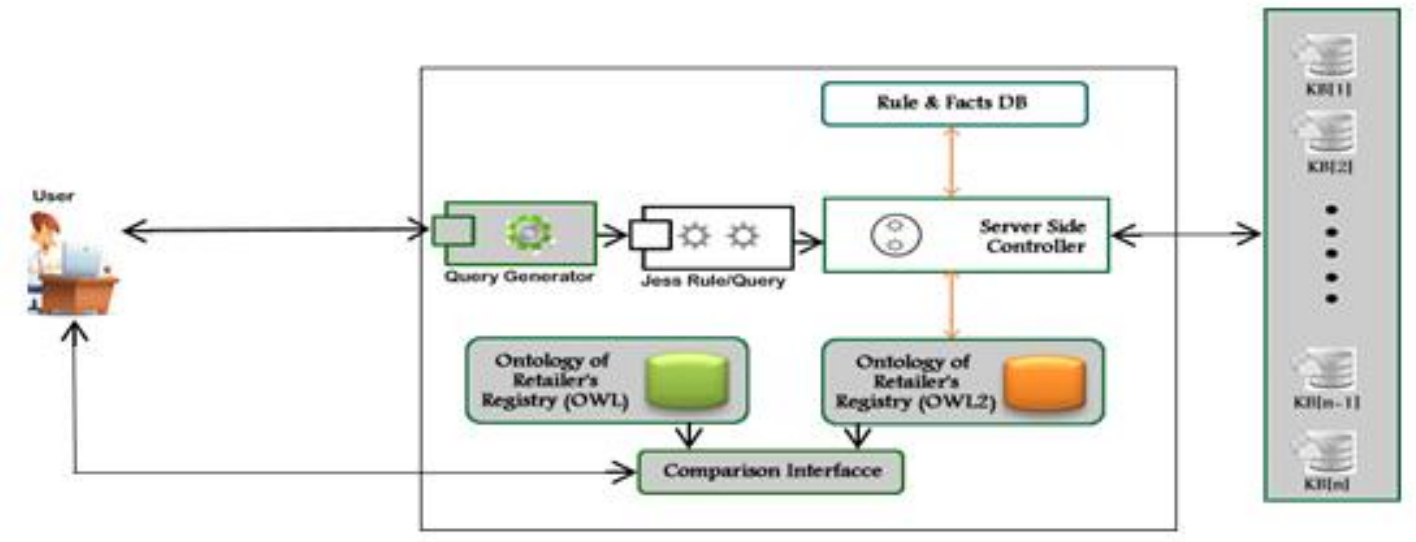

Figure 1: Architecture of the Proposed Ecommerce System

Rules are necessary in this system. The essence of the relevance of incorporating a rule set into this system is to easily entail supplementary information that is not originally modelled in the ontological knowledgebase. These rules are invoked against the knowledgebase. Note that this system does not depend only on the specified rule set. It also works with a reasoner. Reasoners like Pellet, Racer and Fact ++ are useful in inferring what is not readily available in the ontological database. In this research, Pellet 2.2 is being used for the reasoning task.

This proposed system uses two ontologies: the core ontology and the retailer or dealer ontology. The core ontology serves as a registry for all registered retailers or dealers on the web page. This registry is usually looked up en-route in search of information about products online.

This module handles the logical coordination of the entire system. All necessary call to other components and sub modules are being coordinated by this controller. The controller also controls access to the retailer products ontology or database. This enables it to present the user or the online searcher with the result of the search being carried out.

This interface was developed to show the results of a product search in [4] model that was developed with OWL and product search using the proposed model that was developed with OWL2 and the results are being compared in this interface.

\section{THE E-COMERCE ONTOLOGY DEVELOPMENT}

The ecommerce ontology consists of the central registry ontology (also known as core ontology) and the retailer registry ontology. They are discussed in details in this section, and are visually illustrated.

\subsection{The Core Ontology and Retailer's Registry}

Figure 2 illustrates the ontological knowledge base designed for this thesis. It consists of the following concepts: Dealers (which in turn consists of the following subclasses: ProducerDealer, RetailerDealer, and WholeSaleDealer), Location, Person (which also consists of the following: Buyer, Seller), Product, ProductCategory (Automobile, Electronics\&Computer, and Wears), and TradesOrg. The visual display of the core ontology is shown in Figure 2. 


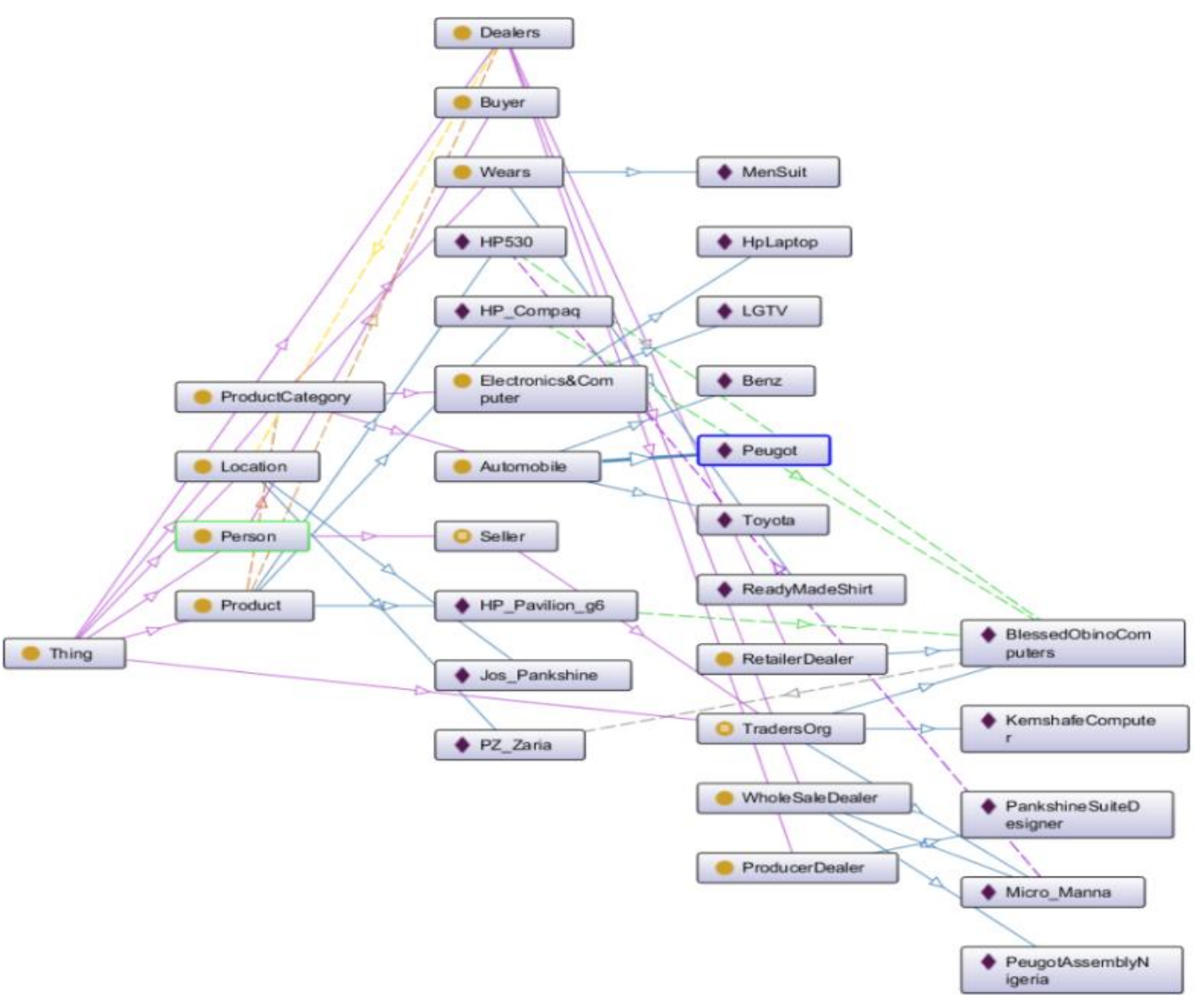

Figure 2: The Core Ontology/Retailer's Registry

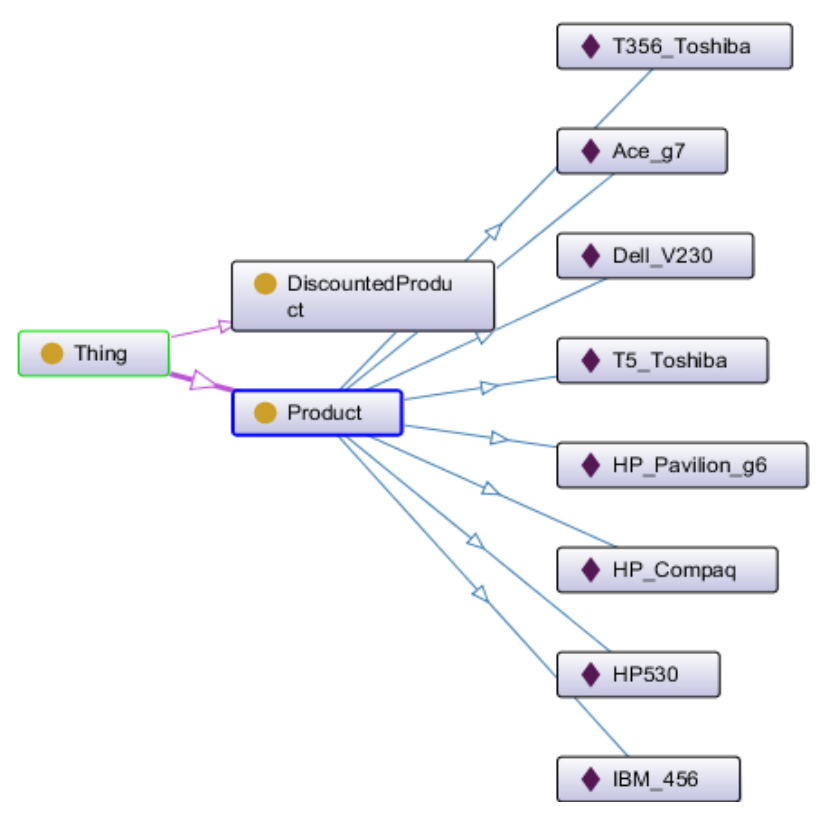

Figure 3: Retailer's Ontology

\subsection{Distributed Retailer Knowledgebase}

Figure 3 is a complete illustration of all the concepts and relations used to model the retailer ontology. This ontology consists of available products that a particular retailer sells. For example, when a retailer markets its presence on the core ontology, the person searching for products online will be redirected back to the retailer ontology for specific search of product.

Algorithm of the Proposed System

Step1: Connect to server from client side

Step2: Let $\mathrm{S}=$ User search word

Step3: Array swords = tokenized from users search word 
Step4: For each sword as w

If $w$ is in ontology

$$
\text { Add } w \text { to queryString }
$$

else

$$
\text { Remove from swords }
$$

Step5: Construct SWRL+SQWRL query from queryString

Step6: Execute query

Step7: Iterate through result and display

\section{IMPLEMENTATION AND RESULT}

In this section, the implementation of the proposed model will be presented. Also, an application that enables the comparison of the ontology of the [4] and that of the ontology proposed by this model is presented.

\subsection{The Comparison Ontology of [4] and this Proposed Ontology}

To implement the work, we had to compare our proposed model using OWL2 with the model earlier used using OWL by [4]. See Figure 4 for the comparison model. This model is designed to allow the two ontologies (modeled with OWL1 and OWL2) to be uploaded for onward comparison. The implication of this comparison is to enable same query be executed observe the two ontologies and observed the number of records that can be retrieved from the ontologies each. And by so doing, modeling of domain knowledge would be preferred to OWL (or OWL1). Note that henceforth, OWL will be used to refer to OWL1.

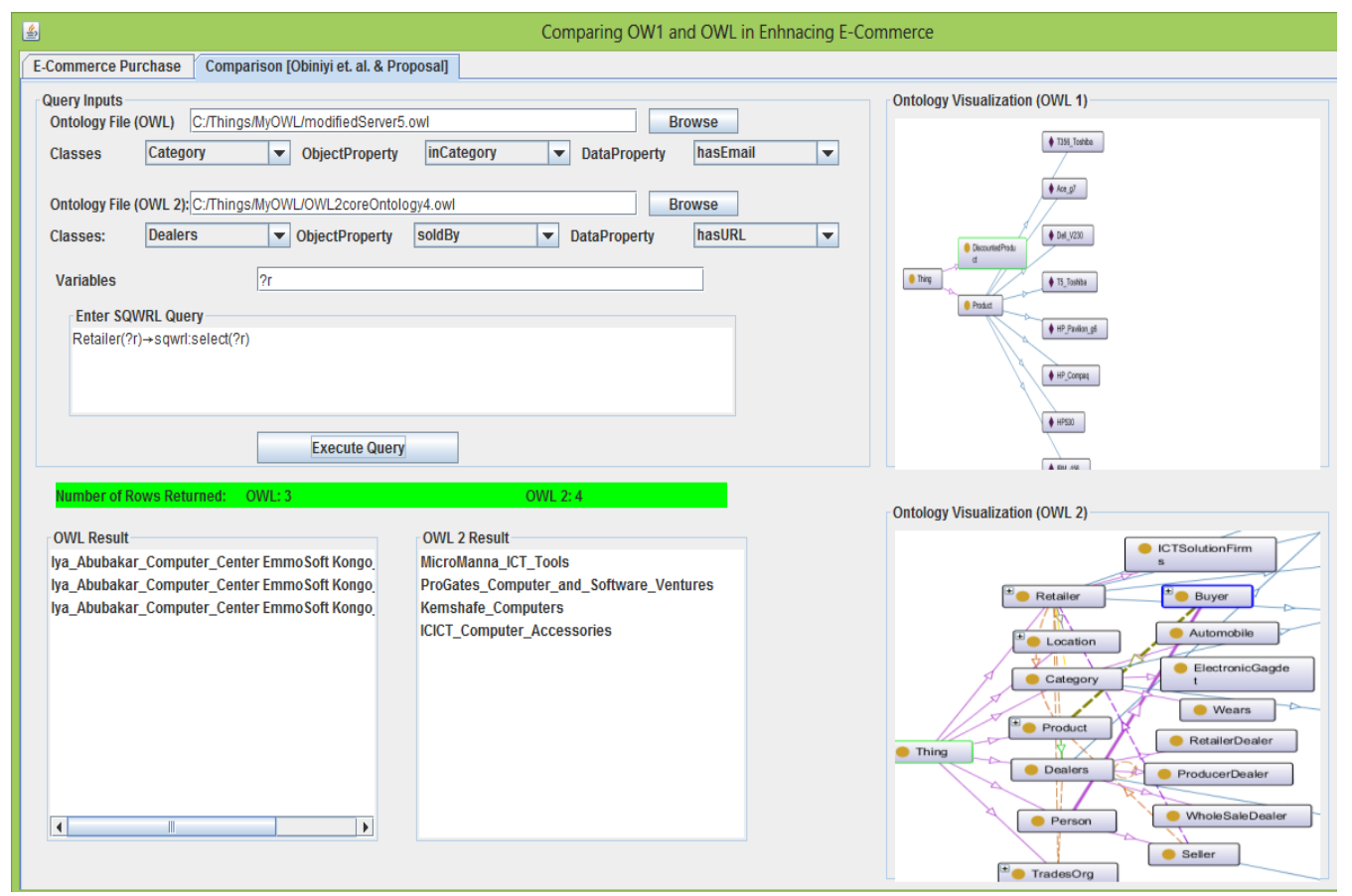

Figure 4: The OWL1 and OWL2 comparison Interface

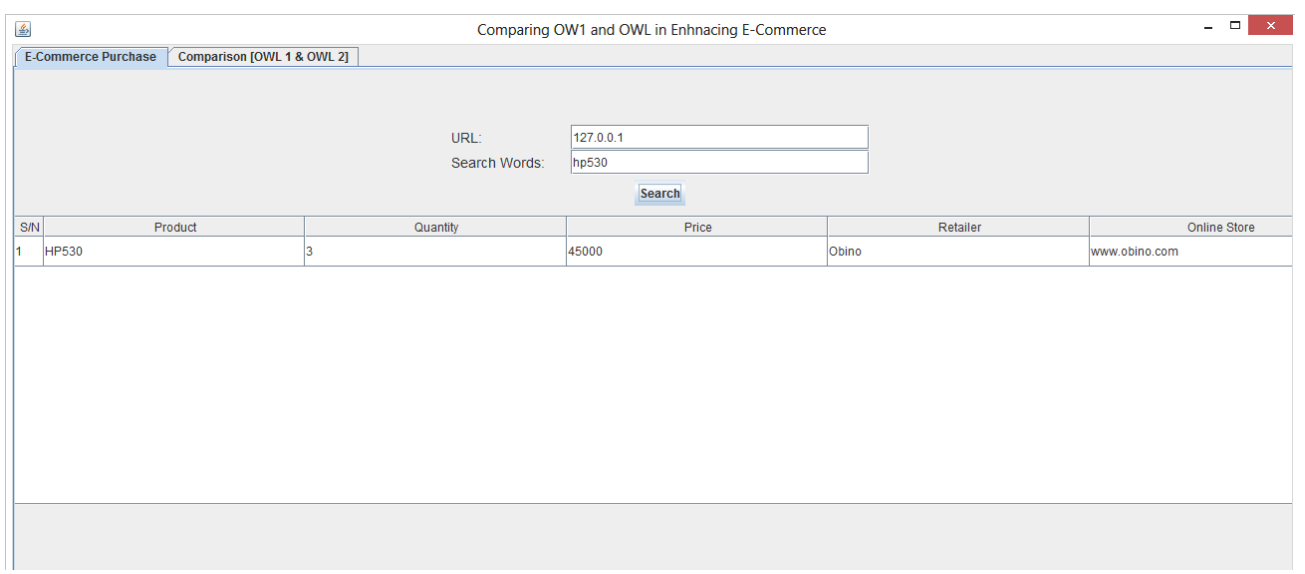

Figure 5 The E-Commerce graphical users Interface

\subsection{The E-Commerce Application}

Again developing an e-commerce application was one of our principal objectives in the entire work. This was achieving also here. Running the Main.java file launches the application for the e-commerce as well, see e-commerce graphical user interface below in Figure 5.

\subsection{Results and Discussion}

Few queries are generated and tested against the similar OWL 
and OWL2 ontologies so as to observe and compare the number of search the could make. Tables 1 and Table 2 gives some comparison figures observed in this research.

Table 1: Generated queries and their descriptions

\begin{tabular}{|c|c|c|}
\hline $\begin{array}{l}\text { Quer } \\
\text { y ID }\end{array}$ & Query & Description \\
\hline $\begin{array}{l}\text { Quer } \\
y 1\end{array}$ & $\begin{array}{l}\text { Reatiler }(? r) \\
\rightarrow \text { sqwrlb: select }(? r)\end{array}$ & $\begin{array}{l}\text { Selects all } \\
\text { available retailers } \\
\text { from the ontology }\end{array}$ \\
\hline $\begin{array}{l}\text { Quer } \\
\text { y2 }\end{array}$ & $\begin{array}{l}\text { Reatiler }(? r), \text { Product } \\
\text { belongsTo }(? r, ? p) \\
\rightarrow \text { sqwrlb: } \operatorname{select}(? r)\end{array}$ & $\begin{array}{l}\text { Selects retailers } \\
\text { who belongs to a } \\
\text { related group } \\
\text { product dealers }\end{array}$ \\
\hline $\begin{array}{l}\text { Quer } \\
\text { yID }\end{array}$ & Query & Description \\
\hline $\begin{array}{l}\text { Quer } \\
\text { y3 }\end{array}$ & $\begin{array}{l}\text { Reatiler }(? r), \\
\text { soldBy }(H P 530, \\
? r) \rightarrow \\
\text { sqwrlb: select }(? r)\end{array}$ & $\begin{array}{lr}\text { Selects retailers } \\
\text { who sells a } \\
\text { particular product }\end{array}$ \\
\hline $\begin{array}{l}\text { Quer } \\
\text { y4 }\end{array}$ & $\begin{array}{l}\text { Reatiler }(? r), \text { Location } \\
\text { locatedAt }(? r, ? l) \\
\rightarrow \text { sqwrlb: select }(? r)\end{array}$ & $\begin{array}{l}\text { How retailers are } \\
\text { located in a given } \\
\text { location }\end{array}$ \\
\hline $\begin{array}{l}\text { Quer } \\
y 5\end{array}$ & $\begin{array}{l}\text { Reatiler }(? r), \text { Seller }(? \\
\text { sellsFor }(? s, ? r) \\
\rightarrow \text { sqwrlb: select }(? r)\end{array}$ & $\begin{array}{l}\text { Does a retailer it } \\
\text { have } \\
\text { sellers? }\end{array}$ \\
\hline
\end{tabular}

Table 2: Generated Queries and their Performances on two differently Modeled Ontologies

\begin{tabular}{|l|l|l|}
\hline Query ID & {$[4]$ System } & $\begin{array}{l}\text { Proposed } \\
\text { System }\end{array}$ \\
\hline Query1 & 3 & 4 \\
\hline Query2 & 1 & 2 \\
\hline Query3 & 1 & 1 \\
\hline Query4 & 3 & 4 \\
\hline Query5 & 0 & 3 \\
\hline
\end{tabular}

The graph shown by Figure 6 is a graphical illustration of how an E-Commerce application will greatly improve its product search should the knowledgebase be developed in an expressive way.

Objective number three of this work is to enhance the efficiency of B2C by implementing the Ontology using OWL2 rather than OWL we saw the design of the ontology in chapter three and implements how these ontologies are being accessed. The implementation of this application was designed in such a way that when the user search request is being translated to SWRL, a corresponding SQWRL query is generated for the OWL1ontology. These two corresponding queries are then run to observe their various outputs and then evaluated if truly using SQWRL to query OWL2 file used to model information in $\mathrm{B} 2 \mathrm{C}$ environment, is more efficient than SQWRL to query and ontology using OWL1. The two queries are channeled to different files and then compared. Figure 4 captures the result after the corresponding query was run against the different ontologies.

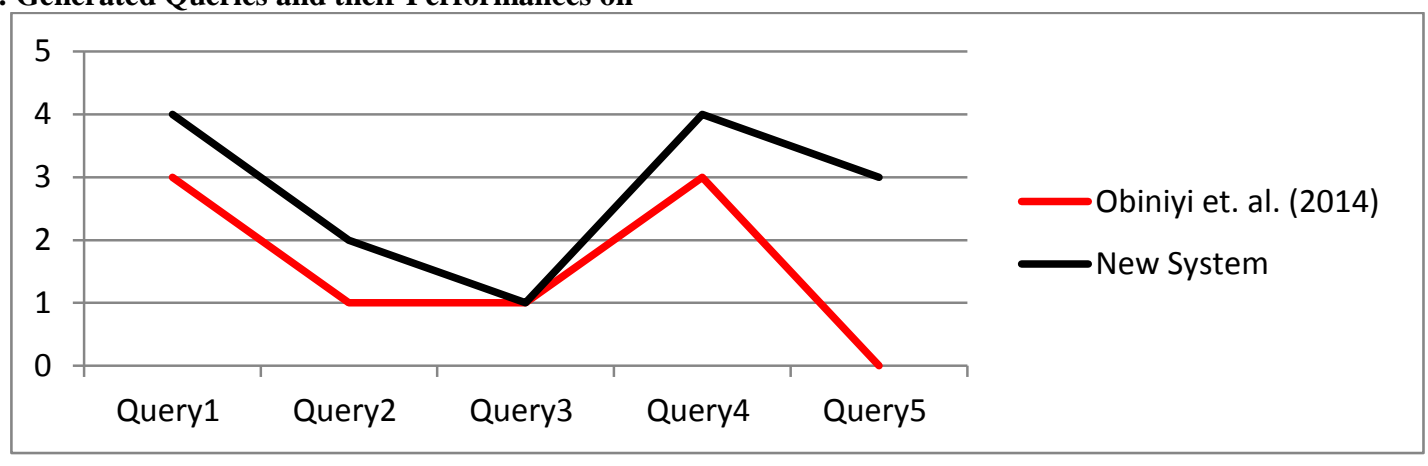

Figure 6: A graphical illustration of efficiency modeling E-Commerce knowledgebase with OWL2 over OWL

Looking closely at Figure 4, SQWRL with OWL1 returns no result while SQWRL with OWL2 ontology returns 1 rows of result. It obvious that SQWRL querying OWL2 ontology is more efficient than SQWRL when used against OWL with such constructs described in chapter two.

\section{CONCLUSION}

The main contribution of this thesis is the improvement of the results or information that would be retrieved from a B2C (an aspect of E-commerce) Web applications or databases only if a better data modeling language as well as when a better query language is chosen. To conclude, this research have shown that OWL2 constructs that has better expressive and intuitive power proves OWL2 to be more expressive than using OWL or RDF/RDFS to model or create ontologies. In a similar vein, we saw that SQWRL is more efficient when used with OWL2 than when used with OWL- especially when some more powerful OWL2 constructs are used. We showed that B2C search will be better if the aforementioned technologies are used. Finally an ontological Ecommerce application was developed to show the use of these Semantic Web technologies.

\section{REFERENCES}

[1] Web 1.0 vs Web 2.0 vs Web 3.0 vs Web 4.0 vs Web 5.0(WEB1.0)(2016): - A bird's eye on the evolution 
and definition Retrieved on 16-6-2016 from https://flatworldbusiness.wordpress.com/flateducation/previously/web-1-0-vs-web-2- 0-vs-web-3-0a-bird-eye-on-the-definition/

[2] Zhang L. and Huang W. (2009). A Framework for an Ontology-based E-commerce Product Information Retrieval System. Journal Of Computers, Academy Publisher 4(6), pp 439-440

[3] Kerie, A. (2014). Engineering Semantic Web for ECommerce Business Intelligence: A Bilingual EEPS Ontology Model. A master thesis submitted to the school of Graduate studies of Arba Minch University.

[4] Oyelade O., Junaidu S., and Obiniyi A. (2014).Semantic Web Framework for E-Commerce Based on OWL. IJCSI International Journal of Computer Science. Issues, Vol. 11, Issue 3, No 2, May 2014 ISSN (Print): 1694-0814 | ISSN (Online): 1694-0784.www.IJCSI.org

[5] Obiniyi A.A, Oyelade O.N and Junaidu S.B (2014). Enhancing Reasoning and Retrieval Performance on $O W L$ using SQWRL. International Journal of Engineering Research \& Technology (IJERT), Vol. 3 Issue 2, February.

[6] Yao W., Basu S., Li J., and Stephenson B. (2012). Modeling and Configuration of Process Variants for Onboarding Customers to IT Outsourcing. HP Laboratories HPL- 2012-157, Hewlett-Packard Development Company, L.P.

[7] Jayaprabha P, and Saradha A (2010),Semantic Integration Of Heterogeneous Web Data For Tourism
Domain Using Ontology Based Resource Description Language. Journal of Computer Applications, 3(3),pp4-5.

[8] Antoniou, G., and Frank V. H. (2004). Semantic Web Primer, MIT Press, Cambridge.

[9] Berners-Lee, T. (1994). Weaving the Web, The MIT Press, Cambridge

[10] Berners-Lee, T., Hendler, J. and Lissila, O. (2001. The Semantic Web. [Online]. Retrieved on 10-5-2016 from http://sciam.com/article.cfm?articleID=00048144-10D21C70-84A9809EC588EF21.

[11] Cuenca B., Horrocks I., Motik B., Parsia B., PatelSchneider P., and Sattler U (2008). OWL 2: The Next Step for $O W L$

[12] Bizer, C., F., and Heath, T., T., Berners-Lee, T., (2009). Linked Data- the Story so Far, International Journal on Semantic Web and Information System (IJSWIS,) Vol. 5(3), pp. 1-23.

[13] Gruber, T., (1993). A Translation Approach to Portable Ontologies, Knowledge Acquisition, 5( 2), pp. 199-220. Management

[14] OWL 2 Web Ontology Language Document Overview (Second Edition).Retrieved on 16-6-2016 from https://www.w3.org/2007/OWL/wiki/Document_Overvie w.

[15] OWL 2 Web Ontology Languages New Features and Rationale (Second Edition) W3C Recommendation 11 December 2012. Retrieved on 17-6-201 from https://www.w3.org/TR/owl2-new-features/. 